\title{
Endoscopic ultrasonography-identified celiac adenopathy remains a poor prognostic factor despite preoperative chemoradiotherapy in esophageal adenocarcinoma
}

\author{
S. Chris Malaisrie, MD, a,e Wayne L. Hofstetter, MD, ${ }^{a}$ Arlene M. Correa, $\mathrm{PhD}^{\text {a }}$ Jaffer A. Ajani, MD, ${ }^{\mathrm{b}}$ \\ Ritsuko R. Komaki, MD, ${ }^{c}$ Zhongxing Liao, MD, ${ }^{c}$ Alexandria Phan, ${ }^{b}$ David C. Rice, MD, ${ }^{a}$ Ara A. Vaporciyan, MD, \\ Garrett L. Walsh, MD, ${ }^{a}$ Sandeep Lahoti, MD, ${ }^{d}$ Jeffrey H. Lee, MD, ${ }^{d}$ Robert Bresalier, MD, ${ }^{d}$ Jack A. Roth, MD, ${ }^{a}$ \\ and Stephen G. Swisher, MD
}

From the Departments of Thoracic and Cardiovascular Surgery, ${ }^{\mathrm{a}}$ Gastrointestinal Medical Oncology, ${ }^{\mathrm{b}}$ Radiation Oncology, ${ }^{\mathrm{c}}$ and Gastrointestinal Medicine and Nutrition, ${ }^{\mathrm{d}}$ The University of Texas M.D. Anderson Cancer Center, and the Division of Cardiothoracic

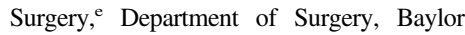
College of Medicine, Houston, Tex.

Support for this study was obtained in part from the George Sweeney Esophageal Research Fund.

Read at the Eighty-fifth Annual Meeting of The American Association for Thoracic Surgery, San Francisco, Calif, April 10-13, 2005.

Received for publication April 4, 2005; revisions received Aug 19, 2005; accepted for publication Aug 30, 2005.

Address for reprints: Stephen G. Swisher, MD, Professor of Surgery, The University of Texas M.D. Anderson Cancer Center, Department of Thoracic and Cardiovascular Surgery, Deputy Chairperson, Academic Affairs, 1515 Holcombe Blvd, Box 445, Houston, TX 77030 (E-mail: sswisher@ mdanderson.org).

J Thorac Cardiovasc Surg 2006;131:65-72

$0022-5223 / \$ 32.00$

Copyright () 2006 by The American Association for Thoracic Surgery

doi:10.1016/j.jtcvs.2005.08.037
Objective: We reviewed our experience with preoperative chemoradiotherapy in patients with adenocarcinoma of the distal esophagus and pretreatment endoscopic ultrasonography-identified celiac adenopathy.

Methods: One hundred eighty-six patients with adenocarcinoma of the distal esophagus were staged with endoscopic ultrasonography before treatment from 1997 through 2004. All patients were treated with concurrent chemoradiotherapy (CRT group) and surgical intervention or induction chemotherapy followed by concurrent chemoradiotherapy (C $\rightarrow$ CRT group) and surgical intervention. Survival analysis (excluding operative mortality) evaluated various pretreatment factors.

Results: Multivariable Cox regression analysis showed that pretreatment endoscopic ultrasonography-identified celiac adenopathy was a significant predictor of decreased long-term survival $(P=.03)$. Median and 3-year survivals were 49 months and 54\% in the endoscopic ultrasonography-identified cN0 M0 group $(\mathrm{n}=65), 45$ months and $56 \%$ in the endoscopic ultrasonography-identified cN1 M0 group ( $\mathrm{n}=$ 96), and 19 months and $12 \%$ in the endoscopic ultrasonography-identified celiac adenopathy (cM1a) group $(\mathrm{n}=18 ; P=.03)$. Increased systemic relapse was noted in the endoscopic ultrasonography-identified cMla group ( $44 \%$ vs $22 \%, P=.07)$. The only factor associated with increased survival in the endoscopic ultrasonographyidentified cM1a group (27 vs 15 months, $P=.02$ ) was the addition of induction chemotherapy before concurrent chemoradiotherapy and surgical intervention.

Conclusions: Endoscopic ultrasonography-identified celiac adenopathy in patients with adenocarcinoma of the distal esophagus conveys a poor prognosis despite preoperative chemoradiotherapy. These patients should be stratified in future multimodality trials. The investigation of induction chemotherapy before concurrent chemoradiotherapy might be warranted in this high-risk group of patients.

$\mathrm{D}$ istal esophageal adenocarcinoma associated with celiac lymph node metastasis has been associated with a high risk of recurrence and poor overall survival after esophagectomy. ${ }^{1}$ Clinical staging with the use of endoscopic ultrasonography (EUS) has permitted the identification of patients with celiac adenopathy before the initiation of treatment with an accuracy of 90\% (and up to $100 \%$ when fine-needle aspiration is added). ${ }^{2}$ Efforts to identify this cohort of patients by means of noninvasive testing has resulted in reports associating EUSidentified celiac adenopathy (cM1a) with poor survival in patients treated with surgical intervention alone, ${ }^{3}$ suggesting that patients with EUS-identified celiac adenopathy could be candidates for further treatment. Although no clear treatment strategy has emerged for patients with EUS-identified celiac adenopathy, the use of 


\section{Abbreviations and Acronyms \\ $\mathrm{cM1a}=$ celiac adenopathy \\ EUS $=$ endoscopic ultrasonography}

preoperative chemoradiotherapy has been evaluated in several centers to treat patients with locally advanced esophageal cancer. ${ }^{4,5}$ We therefore evaluated our experience with preoperative chemoradiotherapy in patients with adenocarcinoma of the distal esophagus and EUS-identified celiac adenopathy at initial presentation.

\section{Patients and Methods \\ Patients}

From a prospectively maintained database at the University of Texas M.D. Anderson Cancer Center, 544 patients were identified who were treated with surgical intervention from January 1997 through March 2004. Of these patients, 241 were excluded because they did not receive pretreatment EUS at our institution, 50 were excluded because they did not have adenocarcinoma of the distal esophagus, 3 were excluded because of known distant metastasis, 49 were excluded because they did not undergo preoperative chemoradiotherapy, and 15 were excluded because they did not undergo primary esophagectomy (redo esophagectomy, salvage esophagectomy, or exploration). The final cohort was 186 patients with primary adenocarcinoma of the distal esophagus, defined as distal to the inferior pulmonary vein up to and including the gastroesophageal junction, who underwent pretreatment EUS and received preoperative chemoradiotherapy followed by primary esophagectomy. Informed consent was obtained, and the study was performed with approval from the M.D. Anderson Cancer Center Review Board. Pretreatment EUS stratified these patients into 3 groups: the node-negative group ( $\mathrm{cN} 0 \mathrm{M} 0, \mathrm{n}=71$ ), the regional adenopathy group $(\mathrm{cN} 1 \mathrm{M} 0, \mathrm{n}=97)$, and the celiac adenopathy group (cM1a, $\mathrm{n}=18)$.

\section{Preoperative Assessment}

All patients were confirmed to have adenocarcinoma of the distal esophagus with esophagoscopy and biopsy. EUS and computed tomography of the chest and abdomen were performed to determine pretreatment clinical stage. Pretreatment positron emission tomography was performed in 83 (45\%) patients. Lymph nodes identified by means of EUS were characterized by the following criteria: round shape, hypoechoic texture and sharp margin, or size greater than $10 \mathrm{~mm}$. Any lymph node found within $1 \mathrm{~cm}$ of the celiac axis manifesting any one or more of the above criteria was considered to have metastatic involvement. Patients were assessed by a multidisciplinary team (thoracic surgeon, medical oncologist, radiation oncologist, gastroenterologist, and radiologist) to determine tumor resectability and physiologic ability to tolerate preoperative chemoradiotherapy and surgical intervention.

\section{Preoperative Chemoradiotherapy}

All patients received neoadjuvant therapy and were treated on institutional protocols with induction chemotherapy followed by concurrent chemoradiotherapy or off protocol with concurrent chemoradiotherapy alone. Patients treated on institutional protocols received up to 2 cycles of irinotecan $\left(45 \mathrm{mg} / \mathrm{m}^{2}\right)$, docetaxel (Taxotere; $33 \mathrm{mg} / \mathrm{m}^{2}$ ), and 5 -fluorouracil $\left(2 \mathrm{~g} / \mathrm{m}^{2}\right.$ as a 24 -hour infusion). This was followed by concurrent chemoradiotherapy (50.4 Gy in 28 fractions) and irinotecan $\left(30 \mathrm{mg} \cdot \mathrm{m}^{-2} \cdot \mathrm{wk}^{-1}\right.$ for 5 weeks), docetaxel $\left(20 \mathrm{mg} \cdot \mathrm{m}^{-2} \cdot \mathrm{wk}^{-1}\right.$ for 5 weeks), and 5 -fluorouracil $\left(300 \mathrm{mg} \cdot \mathrm{m}^{-2} \cdot \mathrm{d}^{-1}\right)$. Patients treated off protocol received concurrent radiotherapy (50.4 Gy in 28 fractions) and chemotherapy, consisting of either cisplatin and 5-fluorouracil or paclitaxel and carboplatin.

\section{Surgical Intervention}

Operative resection was performed approximately 4 to 6 weeks after completion of the preoperative regimen. Four operative approaches were used. The Ivor Lewis esophagectomy used a right thoracotomy and laparotomy for subtotal esophagectomy and proximal gastrectomy with a mediastinal and abdominal lymphadenectomy. The McKeown transthoracic esophagectomy involved resection of the entire thoracic esophagus with mediastinal and abdominal lymphadenectomy, followed by a cervical anastomosis. The transhiatal resection involved a subtotal esophagectomy with abdominal and lower thoracic lymphadenectomy through an abdominal incision, followed by a cervical anastomosis with the conduit in the posterior mediastinal position. The minimally invasive esophagectomy involved thoracoscopic and laparoscopic mobilization of the esophagus and stomach, followed by an open cervical anastomosis. Lymph node dissections were performed in similar fashion to open procedures in abdominal and thoracic compartments. Thoracic lymphadenectomy included level 7 (subcarinal), level 8 (periesophageal), and level 9 (inferior pulmonary ligament) nodes. Abdominal lymphadenectomy included level 16 (perigastric), level 17 (left gastric), and level 18 (hepatic artery) nodes. Level 20 (celiac) nodes were taken separate from the specimen when they were found to be suspicious for malignant involvement. All patients received a tube jejunostomy for temporary enteral nutrition.

\section{Follow-up}

Patients were assessed at 1,3,6, and 12 months and then every 6 months thereafter. Median potential follow-up was 32 months (range, 1-87 months) in the cN0 M0 group, 34 months (range, 1-86 months) in the $\mathrm{cN} 1 \mathrm{M} 0$ group, and 30 months (range, 1-77 months) in the cM1a group.

\section{Statistical Analysis}

Patient characteristics were compared by using analysis of variance, the Pearson $\chi^{2}$ test, or the Fisher exact test where appropriate. Survival distributions were graphically displayed by using the Kaplan-Meier method. Differences in survival distributions were assessed by using the log-rank test. Overall survival was calculated from the date of surgical intervention to the date of death or the date of last follow-up. Disease-free survival was similarly calculated from the date of surgical intervention to the date of disease recurrence or death. Analysis of survival excluded operative mortality defined as death within 30 days after the operation or death during the same hospitalization. Univariable Cox regression analysis, including only preoperative patient factors, was performed to 
TABLE 1. Pretreatment characteristics according to EUS-identified node status

\begin{tabular}{|c|c|c|c|c|}
\hline EUS-identified node status & cNO MO (n = 71) & cN1 Mo (n = 97) & cM1a $(n=18)$ & $P$ value \\
\hline Age, y (median [range]) & $57(23-76)$ & $61(38-79)$ & $62(41-77)$ & $.22^{*}$ \\
\hline \multicolumn{5}{|l|}{ Sex } \\
\hline Male & $65(92 \%)$ & $92(95 \%)$ & $17(94 \%)$ & \multirow[t]{2}{*}{.75} \\
\hline Female & $6(8 \%)$ & $5(5 \%)$ & $1(6 \%)$ & \\
\hline \multicolumn{5}{|l|}{ Tumor stage } \\
\hline cT1 & $0(0 \%)$ & $1(1 \%)$ & $0(0 \%)$ & \multirow[t]{4}{*}{.07} \\
\hline cT2 & $16(23 \%)$ & $7(7 \%)$ & $2(11 \%)$ & \\
\hline cT3 & $54(76 \%)$ & $85(88 \%)$ & $16(89 \%)$ & \\
\hline cT4 & $1(1 \%)$ & $4(4 \%)$ & $5(3 \%)$ & \\
\hline Tumor size, $\mathrm{cm}$ (mean $\pm \mathrm{SD}$ ) & $4.1 \pm 1.7$ & $5.2 \pm 2.7$ & $6.0 \pm 2.9$ & .004 \\
\hline \multicolumn{5}{|l|}{ Histologic grade } \\
\hline Well & $4(6 \%)$ & $3(3 \%)$ & $0(0 \%)$ & \multirow[t]{4}{*}{.052} \\
\hline Moderately & $36(51 \%)$ & $33(34 \%)$ & $7(39 \%)$ & \\
\hline Poor & $23(32 \%)$ & $53(55 \%)$ & $11(61 \%)$ & \\
\hline Unspecified & $8(11 \%)$ & $8(8 \%)$ & $0(0 \%)$ & \\
\hline Barrett's mucosa & $27(38 \%)$ & $37(38 \%)$ & $7(39 \%)$ & .99 \\
\hline \multicolumn{5}{|l|}{ Preoperative therapy } \\
\hline $\mathrm{C} / \mathrm{RT}$ & $26(37 \%)$ & $34(35 \%)$ & $9(50 \%)$ & \multirow[t]{2}{*}{.48} \\
\hline $\mathrm{C} \rightarrow \mathrm{C} / \mathrm{RT}$ & $45(63 \%)$ & $63(65 \%)$ & $9(50 \%)$ & \\
\hline
\end{tabular}

EUS, Endoscopic ultrasonography; $S D$, standard deviation; $C / R T$, concurrent chemoradiotherapy; $C \rightarrow C / R T$, induction chemotherapy followed by concurrent chemoradiotherapy. $* P$ value calculated from mean ages.

identify factors associated with survival. A multivariable Cox regression model was then performed. A stepwise variable selection procedure was used to identify variables most associated with survival in a multivariable context $(P<.10)$. Data analysis was performed by our departmental biostatistician (A.M.C.) using the Statistical Package for Social Sciences (SPSS version 11.5.2.1; SPSS Inc, Chicago, Ill).

\section{Results}

\section{Demographics and Preoperative Characteristics}

All of the patients included in this analysis underwent preoperative chemoradiotherapy and surgical resection and were identified as having adenocarcinoma of the distal esophagus. Pretreatment EUS stratified patients according to nodal involvement into 3 groups: the node-negative group ( $\mathrm{cN} 0 \mathrm{M} 0, \mathrm{n}=71$ ), the regional adenopathy group (cN1 M0, $\mathrm{n}=97$ ), and the celiac adenopathy group (cM1a, $\mathrm{n}=18$ ). The groups did not differ with respect to age, sex, tumor stage, histologic grade, or the presence of Barrett's mucosa (Table 1).

There was a significant difference in tumor size, as determined by pretreatment esophagogastroduodenoscopy, ranging from $4.1 \mathrm{~cm}$ in the node-negative group to $6.0 \mathrm{~cm}$ in the celiac adenopathy group $(P=.004$, Table 1$)$. Higher tumor stage and worse tumor differentiation were observed in the more advanced groups, but these differences were not statistically significant. The preoperative chemoradiotherapy regimen was similar between groups, with 69 (37\%) patients receiving concurrent chemoradiotherapy and 117 (63\%) patients receiving induction chemotherapy followed by concurrent chemoradiotherapy.

\section{Postoperative Characteristics}

Ivor Lewis esophagectomy with lymph node dissection was the most common operative approach in this study (Table 2). The decision to use the transhiatal or McKeown approach was operator dependent. Operative mortality was not different between patients with $\mathrm{cN} 0 \mathrm{M} 0, \mathrm{cN} 1 \mathrm{M} 0$, or $\mathrm{cM} 1 \mathrm{a}$ disease (overall $2.7 \%, P=.22$ ). The rate of pathologic complete response after preoperative chemoradiotherapy was similar among all 3 lymph node groups (overall 26\%, $P=.65)$. Pathologically confirmed metastasis to the celiac nodes (pM1a) was confirmed in $5(28 \%)$ of 18 patients in the EUS-identified cM1a group after chemoradiotherapy and surgical resection.

We explored the patterns of failure for the individual groups and found that the cMla group demonstrated a greater rate of distant recurrence (8/18 [44\%]) compared with the cN0 M0 (13/71 [18\%]) and cN1 M0 (25/97 [26\%], $P=.07$ ) groups. Both overall survival (Figure 1) and disease-free survival were significantly less in the cM1a group (median overall survival, 19 months) when compared with those of the cM0 groups ( $\mathrm{cN} 0 \mathrm{M} 0$ and $\mathrm{cN} 1 \mathrm{M} 0$ combined: median overall survival, 46 months; $P=.03$ ).

\section{Factors Associated With Survival in Patients With Adenocarcinoma of the Distal Esophagus}

Univariable Cox regression demonstrated that only the presence of EUS-identified celiac adenopathy (cM1a disease) and patient performance status (American Society of Anesthesiologists class) were associated with patient survival (Table 3). Neither the EUS-predicted depth of tumor inva- 
TABLE 2. Postoperative characteristics according to EUS-identified node status

\begin{tabular}{|c|c|c|c|c|}
\hline EUS-identified node status & cNO MO (n = 71) & cN1 Mo $(n=97)$ & cM1a $(n=18)$ & $P$ value \\
\hline Type of operation & & & & .30 \\
\hline Ivor Lewis & $45(63 \%)$ & $72(74 \%)$ & $10(56 \%)$ & \\
\hline Transhiatal & $15(21 \%)$ & $14(15 \%)$ & $5(28 \%)$ & \\
\hline McKeown & $9(13 \%)$ & $11(11 \%)$ & $3(17 \%)$ & \\
\hline Minimally invasive & $2(3 \%)$ & $0(0 \%)$ & $0(0 \%)$ & \\
\hline Operative mortality & $4(6 \%)$ & $1(1 \%)$ & $0(0 \%)$ & .22 \\
\hline pCR & $20(28 \%)$ & $25(26 \%)$ & $3(17 \%)$ & .65 \\
\hline pT & & & & .55 \\
\hline pTO & $21(30 \%)$ & $29(29 \%)$ & $3(16 \%)$ & \\
\hline pT1 & $10(14 \%)$ & $17(18 \%)$ & $4(22 \%)$ & \\
\hline pT2 & $11(15 \%)$ & $13(13 \%)$ & $1(6 \%)$ & \\
\hline pT3 & $29(41 \%)$ & $39(40 \%)$ & $9(50 \%)$ & \\
\hline pT4 & $0(0 \%)$ & $0(0 \%)$ & $1(6 \%)$ & \\
\hline $\mathrm{pN}$ & $22(31 \%)$ & $31(32 \%)$ & $9(50 \%)$ & .29 \\
\hline pM1a & $1(1 \%)$ & $3(3 \%)$ & $5(28 \%)$ & .001 \\
\hline Locoregional recurrence & $7(10 \%)$ & $5(5 \%)$ & $1(6 \%)$ & .53 \\
\hline Distant recurrence & $13(18 \%)$ & $25(26 \%)$ & $8(44 \%)$ & .07 \\
\hline Disease-free survival† & & & & $.03^{*}$ \\
\hline Median (mo) & 46 & 38 & 12 & \\
\hline $3-y$ & $56 \%$ & $51 \%$ & $14 \%$ & \\
\hline Overall survivalt & & & & $.03^{*}$ \\
\hline Median (mo) & 49 & 45 & 19 & \\
\hline $3-y$ & $54 \%$ & $56 \%$ & $12 \%$ & \\
\hline
\end{tabular}

EUS, Endoscopic ultrasonography. *Pvalues are calculated from the $\mathrm{cM} 0$ group (cN0 M0 and $\mathrm{cN} 1 \mathrm{M} 0$ ) versus the $\mathrm{cM} 1 \mathrm{a}$ group. $†$ Median potential follow-up was 32 months. Operative mortality was excluded.

sion (cT stage) nor the EUS-predicted presence of regional adenopathy ( $\mathrm{cN}$ stage) was associated with survival. In addition, the surgical approach was not associated with survival. Stepwise multivariate Cox regression again demonstrated that the presence of EUS-identified celiac adenop-

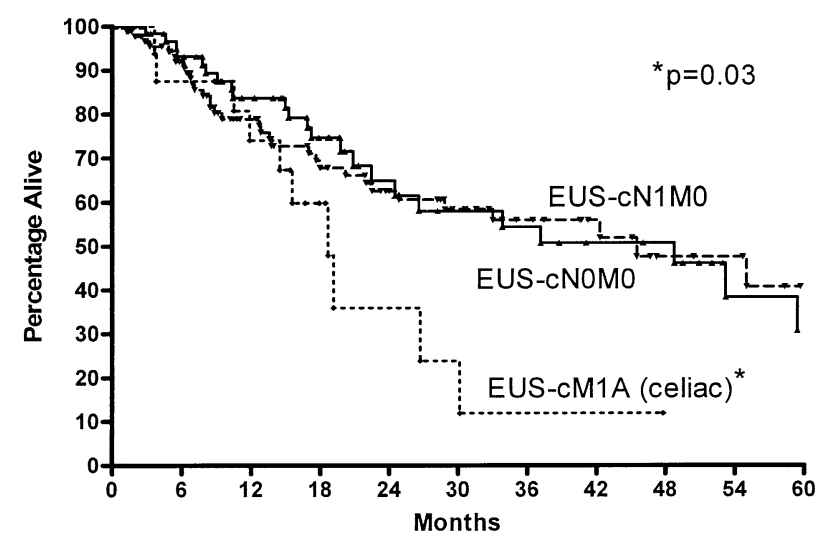

$\begin{array}{lrrrrrr}\text { NOM0: } & 65 & 37 & 16 & 14 & 9 & 4 \\ \text { N1M0: } & 96 & 46 & 30 & 18 & 8 & 4 \\ \text { M1A: } & 18 & 8 & 2 & 1 & 1 & 0\end{array}$

Figure 1. Overall survival of patients with adenocarcinoma of the distal esophagus according to pretreatment and EUS-identified node status (cM1a vs cNO MO and cN1 MO, $P=.03$ ). athy and performance status were independently associated with survival (Table 4). In addition, the multivariate regression showed that tumor depth invasion was also an independent correlate (hazard ratio, 2.44; $P=.045$ ).

\section{Factors Associated With Survival in Patients With cM1a Disease}

In an effort to identify factors associated with improved survival in the patients with EUS-identified celiac adenopathy (cM1a), a separate analysis was performed on this subset of patients. Univariable Cox regression (Table 5) showed that the only factor associated with improved survival in this high-risk subset was the addition of induction chemotherapy before concurrent chemoradiotherapy in the preoperative regimen (Figure 2).

\section{Discussion}

Controversy continues to exist regarding the current treatment and prognosis of patients with celiac axis adenopathy. Most studies have suggested that celiac adenopathy is a poor prognostic factor, especially when treated with surgical intervention alone. ${ }^{1,3,6,7}$ In this study we attempted to evaluate a more homogeneous subpopulation of patients by limiting our study to patients who had undergone pretreatment EUS and had nonmetastatic adenocarcinoma of the distal esophagus or gastroesophageal junction. In an attempt 
TABLE 3. Univariate Cox regression analysis of pretreatment risk factors for long-term survival in patients with adenocarcinoma of the distal esophagus

\begin{tabular}{|c|c|c|c|}
\hline Risk factor & HR & CI & $P$ value \\
\hline Patient age & 1.01 & $0.98-1.03$ & .63 \\
\hline \multicolumn{4}{|l|}{ Sex } \\
\hline Male & 1 & & \\
\hline Female & 0.71 & $0.23-2.29$ & .57 \\
\hline Histologic grade & & & .46 \\
\hline Well differentiated & 1 & & \\
\hline Moderately differentiated & 2.33 & $0.55-9.91$ & .25 \\
\hline Poorly differentiated & 2.49 & $0.59-10.5$ & .21 \\
\hline \multicolumn{4}{|l|}{ T stage (EUS identified) } \\
\hline $\mathrm{cT} 1 / \mathrm{T} 2$ & 1 & & \\
\hline cT3/T4 & 1.62 & $0.77-3.40$ & .20 \\
\hline \multicolumn{4}{|l|}{ Tumor size (EGD identified) } \\
\hline$<5 \mathrm{~cm}$ & 1 & & \\
\hline$\geq 5 \mathrm{~cm}$ & 1.13 & $0.69-1.86$ & .64 \\
\hline \multicolumn{4}{|l|}{ Barret's mucosa* } \\
\hline No & 1 & & \\
\hline Yes & 1.55 & $0.95-2.52$ & .08 \\
\hline \multicolumn{4}{|l|}{ N stage (EUS identified) } \\
\hline cNO & 1 & & \\
\hline $\mathrm{cN1}$ & 1.18 & $0.71-1.97$ & .51 \\
\hline \multicolumn{4}{|l|}{ Node size (EUS identified) } \\
\hline$<1.0 \mathrm{~cm}$ & 1 & & \\
\hline$\geq 1.0 \mathrm{~cm}$ & 1.24 & $0.64-2.39$ & .52 \\
\hline \multicolumn{4}{|l|}{ M stage (EUS identified) } \\
\hline cMO & 1 & & \\
\hline cM1a & 2.01 & $1.06-4.13$ & .04 \\
\hline ASA class ${ }^{-}$ & & & .05 \\
\hline 2 & 1 & & \\
\hline 3 & 1.39 & $0.74-2.62$ & .30 \\
\hline 4 & 4.08 & $1.30-12.85$ & .02 \\
\hline \multicolumn{4}{|l|}{ Preoperative therapy } \\
\hline $\mathrm{C} / \mathrm{RT}$ & 1 & & \\
\hline $\mathrm{C} \rightarrow \mathrm{C} / \mathrm{RT}$ & 0.73 & $0.44-1.22$ & .23 \\
\hline
\end{tabular}

$H R$, Hazard ratio; $\mathrm{Cl}$, confidence interval; EUS, endoscopic ultrasonography; $E G D$, esophagogastroduodenoscopy; $C / R T$, concurrent chemoradiotherapy; $C \rightarrow C / R T$, induction chemotherapy followed by concurrent chemoradiotherapy. *Barrett's mucosa was determined by means of pretreatment EGD and biopsy. $\dagger$ Performance status defined by the American Society of Anesthesiologists (ASA).

to evaluate the effect of preoperative chemoradiation on this subset, only patients who were treated with preoperative chemoradiation and underwent an esophageal resection were included.

The identification of celiac lymph nodes by means of ultrasonography has been found in experienced hands to be quite predictive of pathologic involvement. Results from 2 series demonstrate a sensitivity and specificity with EUS alone of $67 \%$ to $77 \%$ and $85 \%$ to $87 \%$, respectively. Catalano and colleagues ${ }^{10}$ found that preoperative detection of celiac lymph nodes by means of EUS with characteriza-
TABLE 4. Multivariate Cox regression analysis of pretreatment risk factors for long-term survival in patients with adenocarcinoma of the distal esophagus

\begin{tabular}{llll}
\hline Risk factor & HR & CI & P value \\
\hline $\begin{array}{l}\text { T stage (EUS identified) } \\
\text { cT1/T2 }\end{array}$ & 1 & & \\
cT3/T4 & 2.44 & $1.02-5.83$ & .045 \\
M stage (EUS identified) & & & \\
$\quad$ cM0 & 1 & & \\
cM1a & 2.18 & $1.20-4.32$ & .03 \\
Barrett's mucosa* & & & \\
$\quad$ No & 1 & & \\
Yes & 1.64 & $0.99-2.70$ & .055 \\
ASA class $\dagger$ & & & .02 \\
2 & 1 & & .26 \\
3 & 1.43 & $0.76-2.69$ & .01 \\
4 & 4.32 & $1.37-13.65$ & \\
\hline
\end{tabular}

$H R$, Hazard ratio; $\mathrm{Cl}$, confidence interval; $E U S$, endoscopic ultrasonography. *Barrett's mucosa determined by means of pretreatment esophagogastroduodenoscopy. †Performance status defined by the American Society of Anesthesiologists (ASA).

tion by size, shape, echogenicity, and borders had a sensitivity and specificity of $83 \%$ and $98 \%$, respectively, for involvement. False-positive results occurred in only 2 of 145 patients. This has led Eloubeidi and associates ${ }^{8}$ to hypothesize that the mere detection of celiac axis lymph nodes was highly predictive of malignant involvement. Furthermore, celiac lymph nodes larger than $10 \mathrm{~mm}$ have been shown in some studies to be histologically involved $100 \%$ of the time. ${ }^{3}$ In our study celiac adenopathy was not diagnosed pathologically before treatment, and the use of neoadjuvant therapy precluded postoperative confirmation. Despite this potential inaccuracy, the detection of celiac adenopathy on a pretreatment EUS in our study was a strong and independent negative prognostic indicator. Unlike EUS-identified adenopathy in other lymph node locations, such as the mediastinum or paraesophageal region (cN1), celiac adenopathy (cM1a) was a strong predictor of poor outcome, even after preoperative chemoradiation. This is not completely unexpected because previous reports by our group have demonstrated that almost all pretreatment tumor characteristics lose validity after preoperative chemoradiation, with pathologic response and posttreatment pathologic stage becoming the most important predictors of survival. ${ }^{11,12}$ This study suggests that EUS-identified pretreatment celiac adenopathy is such a powerful prognostic factor that even after preoperative chemoradiation, it maintains a negative predictive value.

The reason that celiac adenopathy portends such a poor prognosis might be the systemic nature associated with involvement of the celiac axis. The 2001 American Joint Committee on Cancer $^{13}$ classifies celiac axis involvement as 
TABLE 5. Univariate Cox regression analysis of pretreatment risk factors for long-term survival in patients with cM1a disease

\begin{tabular}{|c|c|c|c|}
\hline Risk factor & HR & Cl & $P$ value \\
\hline Patient age & 1.02 & $0.96-1.08$ & .46 \\
\hline \multicolumn{4}{|l|}{ Sex } \\
\hline Male & 1 & & \\
\hline Female & 0.79 & $0.09-6.32$ & .79 \\
\hline \multicolumn{4}{|l|}{ Histologic grade } \\
\hline Moderately differentiated & 1 & & \\
\hline Poorly differentiated & 1.43 & $0.40-5.13$ & .58 \\
\hline \multicolumn{4}{|l|}{ T stage (EUS identified) } \\
\hline cT1/T2 & 1 & & \\
\hline cT3/T4 & 0.45 & $0.09-2.31$ & .40 \\
\hline \multicolumn{4}{|l|}{ Tumor size (EGD identified) } \\
\hline$<5 \mathrm{~cm}$ & 1 & & \\
\hline$\geq 5 \mathrm{~cm}$ & 3.61 & $0.75-17.43$ & .11 \\
\hline \multicolumn{4}{|l|}{ Barrett's mucosa* } \\
\hline No & 1 & & \\
\hline Yes & 0.97 & $0.18-5.28$ & .97 \\
\hline ASA class $t$ & & & .15 \\
\hline 2 & 1 & & \\
\hline 3 & 1.54 & $0.31-7.57$ & .60 \\
\hline 4 & 20.13 & $0.95-425$ & .05 \\
\hline \multicolumn{4}{|l|}{ Preoperative therapy } \\
\hline $\mathrm{C} / \mathrm{RT}$ & 1 & & \\
\hline $\mathrm{C} \rightarrow \mathrm{C} / \mathrm{RT}$ & 0.19 & $0.04-0.97$ & .04 \\
\hline
\end{tabular}

$H R$, Hazard ratio; $\mathrm{Cl}$, confidence interval; EUS, endoscopic ultrasonography; $E G D$, esophagogastroduodenoscopy; $C / R T$, concurrent chemoradiotherapy; $C \rightarrow C / R T$, induction chemotherapy followed by concurrent chemoradiotherapy. *Barrett's mucosa was determined by means of pretreatment EGD and biopsy. †Performance status identified by the American Society of Anesthesiologists $(A S A)$ criteria.

a metastatic subset, M1a, rather than an N1 designation because of the high likelihood of relapse when surgical intervention alone is used. Steup and coworkers ${ }^{14}$ reported a 5 -year survival of $15 \%$ in patients with gastroesophageal cancer and distant lymph node metastasis (including thoracic and celiac lymph node metastasis). More recently, Eloubeidi and associates ${ }^{3}$ have also demonstrated poor survival in patients with specifically celiac lymph node metastasis when surgical intervention alone is used, with 5-year survivals of $13 \%$ compared with $30 \%$. Although our study was limited by the small number of patients with EUSidentified celiac adenopathy, there appeared to be a tendency toward a higher proportion of patients who relapsed systemically as opposed to locoregionally (Table 2). Systemic relapse for patients with cM1a disease was $44 \%$ versus $18 \%$ and $26 \%$ for patients with $\mathrm{cN} 0 \mathrm{M} 0$ and $\mathrm{cN} 1 \mathrm{M} 0$ disease $(P=.07)$. Interestingly, locoregional relapse was similar between the groups, suggesting that additional improvements in survival for patients with $\mathrm{cM} 1 \mathrm{a}$ disease might need to focus on better systemic control.

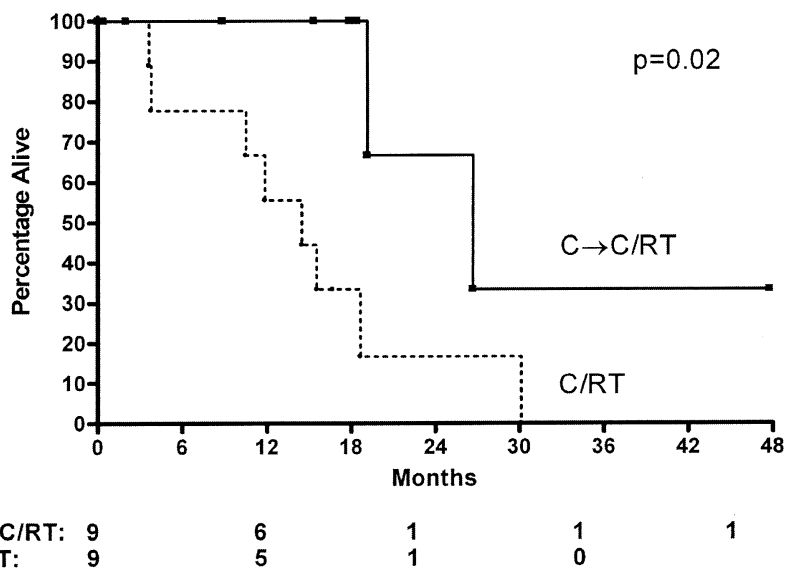

Figure 2. Overall survival of patients with pretreatment, EUSidentified cM1a disease according to preoperative chemoradiotherapy sequence. $C / R T$, Concurrent chemoradiotherapy; $C \rightarrow C / R T$, induction chemotherapy followed by concurrent chemoradiotherapy $(P=.02)$.

Although this study does show that EUS-identified celiac adenopathy is associated with a poor prognosis, the presence of long-term survivors is encouraging. Other studies have confirmed that there are long-term survivors with celiac lymph node disease, and the application of aggressive multimodal therapy, including surgical resection, might be warranted. ${ }^{15}$ In an attempt to identify the subset of patients with cM1a disease who might benefit from preoperative chemoradiation and surgical intervention, multiple factors were analyzed univariably, including age, sex, histologic grade, tumor and nodal size, performance status, and type of preoperative chemoradiation used. Although the small numbers prevent meaningful multivariable analysis, univariable analysis demonstrated improved survival in the group of patients who received additional chemotherapy before concurrent chemoradiation, perhaps because of improved systemic treatment. The addition of induction chemotherapy before concurrent chemoradiotherapy has been shown to improve overall response rates of locally advanced esophageal tumors in several phase II trials, ${ }^{16-18}$ and it is possible that this intensive regimen is beneficial to patients with celiac axis disease. The poor prognosis of this group of patients warrants further evaluation of the role of induction chemotherapy before concurrent chemoradiation in a prospective fashion. Additionally, novel biologic agents might need to be investigated to seek long-term improvement in this difficult group of patients.

It is important to emphasize the limitations inherent in this retrospective study, including the fact that only patients who completed preoperative chemoradiation and surgical intervention were included, leading to bias for higher performance status patients. Although the results of 2 phase II 
trials $^{16,17}$ at our institution showed that $91 \%$ of patients receive their planned dose of chemoradiation, the total percentage in the current study group is unknown because many patients received their preoperative treatment at another institution. Additionally, the small number of patients in the cM1a study group limits the conclusions that can be reached because many factors showed a trend toward being predictors of survival but failed to reach statistical significance. The nonrandomized nature of the study might also have led to selection bias because only patients with higher performance status might have been offered additional induction chemotherapy before concurrent chemoradiation, and finally, the lack of histologic confirmation of celiac lymph node metastasis before the institution of treatment limits our ability to reach definitive conclusions regarding pathologic involvement of the celiac axis.

Despite these limitations, our study confirms and extends previous observations that EUS-identified celiac adenopathy is a poor prognostic factor, even when treated with preoperative concurrent chemoradiation. Identification of this high-risk group before treatment might warrant the inclusion of these patients in novel treatment strategies. Pathologic confirmations of celiac lymph node metastasis by means of EUS-fine-needle aspiration should be included in future studies involving preoperative therapy. Prospective trials might be warranted to evaluate the benefit of aggressive preoperative regimens aimed at improved systemic control for this high-risk group of patients.

We thank Alma Vega and Debbie Smith for help in preparation and review of the manuscript.

\section{References}

1. Christie NA, Rice TW, DeCamp MM, et al. M1A/M1B esophageal carcinoma: clinical relevance. J Thorac Cardiovasc Surg. 1999;118: 900-7.

2. Parmar KS, Zwischenberger JB, Reeves AL, Waxman I. Clinical impact of endoscopic ultrasound-guided fine needle aspiration of celiac axis lymph nodes (M1a disease) in esophageal cancer. Ann Thorac Surg. 2002;73:916-21.

3. Eloubeidi MA, Wallace MB, Hoffman BJ, et al. Predictors of survival for esophageal cancer patients with and without celiac axis lymphadenopathy: impact of staging endosonography. Ann Thorac Surg. 2001; 72:212-20.

4. Malaisrie SC, Untch B, Aranha GV, Mohideen N, Hantel A, Pickleman J. Neoadjuvant chemoradiotherapy for locally advanced esophageal cancer: experience at a single institution. Arch Surg. 2004;139: 532-9.

5. Swisher SG, Holmes EC, Hunt KK, et al. The role of neoadjuvant therapy in surgically resectable esophageal cancer. Arch Surg. 1996; 131:819-25.

6. Akiyama A, Tsurumaru M, Udagawa $\mathrm{H}$, et al. Radical lymph node dissection for cancer of the thoracic esophagus. Ann Surg. 1994;220: 364-73.

7. Bada M, Aikou T, Yoshinaka H, et al. Long-term results of subtotal esophagectomy with three-field lymphadenectomy for cancer of the thoracic esophagus. Ann Surg. 1994;219:310-6.

8. Eloubeidi MA, Wallace MB, Reed CE, et al. The utility of EUS and EUS-guided fine needle aspiration in detecting celiac lymph node metastasis in patients with esophageal cancer: a single-center experience. Gastrointest Endosc. 2001;54:714-9.
9. Kallimanis GB, Gupta PK, al-Kawas FE, Tio LT, Benjamin SB, Bertagnolli MB, et al. Endoscopic ultrasound for staging esophageal cancer, with or without dilation, is clinically important and safe. Gastrointest Endosc. 1995;41:540-6.

10. Catalano MF, Alcocer E, Chak A, et al. Evaluation of metastatic celiac axis lymph nodes in patients with esophageal carcinoma: accuracy of EUS. Gastrointest Endosc. 1999;50:352-6.

11. Chirieac LR, Swisher SG, Ajani JA, et al. Posttherapy pathologic stage predicts survival in patients with esophageal carcinoma receiving preoperative chemoradiation. Cancer. 2005;103:1347-55.

12. Swisher SG, Hofstetter W, Wu TT, et al. Proposed revision of the esophageal cancer staging system to accommodate pathologic response (pP) following preoperative chemoradiation (CRT). Ann Surg. 2005;241:810-20.

13. American Joint Committee on Cancer. AJCC cancer staging manual. 6th ed. New York: Springer-Verlag; 2002. p. 91-8.

14. Steup WH, De Leyn P, Deneffe G, et al. Tumors of the esophagogastric junction: long-term survival in relation to the pattern of lymph node metastasis and a critical analysis of the accuracy or inaccuracy of PTNM classification. J Thorac Cardiovasc Surg. 1996;111:85-95.

15. Frizzell B, Sinha D, Williams T, Reed CE, Sherman CA, Turrisi A. Influence of celiac axis lymph nodes in the definitive treatment of esophageal cancer. Am J Clin Oncol. 2003;26:215-20.

16. Ajani JA, Komaki R, Putnam JB, et al. A three-step strategy of induction chemotherapy then chemoradiation followed by surgery in patients with potentially resectable carcinoma of the esophagus and gastroesophageal junction. Cancer. 2001;92:279-86.

17. Ajani JA, Walsh GL, Komaki R, et al. Preoperative induction of CPT-11 and cisplatin chemotherapy followed by chemoradiotherapy in patients with locoregional carcinoma of the esophagus and gastroesophageal junction. Cancer. 2004;100:2347-54.

18. Swisher SG, Ajani JA, Komaki R, et al. Long-term outcome of phase II trial evaluating chemotherapy, chemoradiotherapy, and surgery for locoregionally advanced esophageal cancer. Int J Radiat Oncol Biol Phys. 2003;57:120-7.

\section{Discussion}

Dr Carolyn E. Reed (Charleston, SC). This study confirms what our group at the Medical University of South Carolina and the Cleveland Clinic have previously shown: M1a disease is a marker of poor clinical outcome. The authors have extended this observation and documented that patients with adenocarcinoma of the distal esophagus and M1a disease identified by means of endoscopic ultrasonography (EUS) have poor outcome, even after aggressive induction therapy.

This article has several limitations. It is retrospective and nonrandomized; the number of patients is small, 18 patients; induction therapy varied; and, most importantly, metastatic celiac nodes were not validated by means of EUS-fine-needle aspiration (FNA). Only 5 of 18 patients had metastatic celiac nodes at the time of surgical intervention. Considering the overall pathologic complete response rate of $26 \%$, it is unlikely that $70 \%$ of patients with M1a disease were downstaged to M0. The authors suggest that patients with clinical M1a disease could benefit from induction chemotherapy, followed by concurrent chemoradiotherapy. This might be a reasonable multicenter approach, but histologic confirmation with EUS-FNA should be required.

I have several questions for the authors. One, why did you not use EUS-FNA? We really do not know how many patients truly had M1a disease.

Dr Malaisrie. We only started using routine EUS-FNA recently, and indeed, 5 of the 18 patients with cM1a disease did undergo FNA before treatment. Because of that small number, we excluded FNA documentation from the analysis, yet despite the 
fact that FNA documentation was not required, endoscopic criteria alone were still a strong negative independent predictor of poor outcome.

Dr Reed. Second, if you used EUS-FNA in your next study, and celiac lymph nodes were still positive after induction therapy, would your group still recommend surgical intervention?

Dr Malaisrie. I think the short answer to that is, yes, they would get treated. This is mainly because most of our patients are treated on protocol, and unless distant disease is present, surgical resection is still performed. If multicenter studies can be performed in the future, perhaps data will one day exist to know how to proceed with this difficult group of patients.

Dr Reed. Three-year survival was the same for clinical N0 M0 and clinical N1 M0 disease. How do you explain this? How did EUS clinical N1 disease correlate with pathologic N1 disease?

Dr Malaisrie. The reason patients with $\mathrm{cN} 1 \mathrm{M} 0$ and $\mathrm{cNO} \mathrm{M} 0$ disease had similar survival might be due in part to the fact that the clinical staging was obtained before pathologic downstaging by preoperative chemoradiation. If patients are simply treated with surgical intervention alone without preoperative chemoradiation, patients with EUS-identified cN0 M0 disease do much better than those with cN1 M0 disease. Despite preoperative chemoradiation, however, patients with cN1 M1a disease do much worse, and I think this is the take-home message: this is a worse group of patients, perhaps with more micrometastatic disease that does not respond well to standard preoperative chemoradiation.

Dr Reed. Finally, did those patients with clinical N1 disease receiving induction chemotherapy followed by chemoradiotherapy, your 3-stage approach, versus chemoradiation also do better clinically?

Dr Malaisrie. In this limited study there was a tendency toward improved survival, but it did not reach statistical significance. In an upcoming presentation at The Western Thoracic Surgical Association meeting, we address this question in more detail in a larger patient population.

Dr Reed. Thank you very much for a very well-presented article.

Dr Mark J. Krasna (Baltimore, $M d$ ). To take a different tack from Dr Reed's question, there are a lot of data that were not mentioned today talking about the outcomes of patients with clinical M1a disease who just go to surgical intervention alone. Presented here at this meeting several years ago by Dr Lerut's group in Belgium and by the group in New York by Rob Korst were actually excellent outcomes of patients who had surgical resection for adenocarcinomas of the gastroesophageal junction and M1a lymph node with survivals that went anywhere from $17 \%$ to as high as $30 \%$. Therefore I do think we need to take a step back. We do need to identify that there can be actually even better survival in this subgroup of patients and that calling their disease metastatic M1a disease was convenient for the staging system but might in fact not be appropriate clinically.

My question, then, comes from a slightly different angle but similar to what Dr Reed is alluding to. Can you identify these patients up front pathologically with, as with Dr Reed and our institution, EUS-FNA, or if we cannot find it by using the FNA, by means of thoracoscopy or laparoscopy? You can then know the status of the patient before undergoing the chemoradiation. There are 2 potential advantages to that. First, you can then allocate the patients for the high-dose chemotherapy followed by the chemoradiotherapy protocol only as appropriate and not subject everyone to the extra morbidity, which I know is significant. Second, you are going to know your actual "n" in the denominator of your study; you are going to know in whom there is some benefit.

Therefore the last question is, of those patients, and I do not think you showed us these data, who did have clinical M1a disease but had persistent M1a disease pathologically after the chemoradiation, was there a difference? Our two prior articles from Maryland, phase I and phase II, looking at chemoradiotherapy followed by surgical intervention, showed that the only important prognosticator was persistent N1 disease, what you are calling M1a disease, after chemoradiotherapy. Was that in fact looked at? It was not listed on your list of variables.

Dr Malaisrie. Thank you, Dr Krasna.

We did look at those data, but because of the small number of patients, we cannot give a definitive answer.

In the 18 patients who we identified with $\mathrm{cM} 1 \mathrm{a}$ disease by means of EUS, at the time of surgical intervention, 4 patients had pathologic M1a disease. Of those 4 patients, 2 are dead at current follow-up. They died at 10 months and 26 months, respectively. The 2 who are alive are still alive at 15 and 18 months.

Dr Krasna. Was that included as one of your variables in the multivariate analysis, response rate? It might turn out to be at least as important as the trimodality versus bimodality approach.

Dr Malaisrie. Perhaps it will be a significant factor, but this will require more patients to confirm.

Dr Stephen D. Cassivi (Rochester, Minn). Thanks. I was very interested in your presentation, and I have a comment that perhaps evolves into a question.

First of all, thank you for bringing another article that underlines the importance of proper staging in esophageal cancer. However, I would underline what Dr Reed and Dr Krasna were mentioning. Specifically, unless you have the FNA, I am not sure we actually know what the true status of those lymph nodes was. You talked about a $30 \%$ false-positive rate in the N1 nodes alone. I think that is brought out by the fact that your median survivals for your patients with $\mathrm{N} 1$ and N0 disease are virtually the same at around 48 months.

What criteria were you using by EUS if you were not using FNA to determine which nodes were positive?

Dr Malaisrie. The 4 criteria that were used by our group were size greater than $1 \mathrm{~cm}$, sharp borders, less echogenicity than the surrounding tissues, and round shape. Our endoscopists consistently used at least one of these criteria to define patients with cM1a disease.

I would like to point out again that this article does give some prognostic information, but I want to be very careful about giving any sort of treatment recommendations because of the small numbers. Our article demonstrates that EUS-identified celiac adenopathy identifies a poor prognostic group that is difficult to care for, even with preoperative chemoradiation. Whether the use of FNA will help to further refine this group in the future remains to be determined by future prospective multicenter studies. 